\title{
Erratum to: Genomic prediction using subsampling
}

Alencar Xavier ${ }^{1}$, Shizhong $\mathrm{Xu}^{2}$, William Muir ${ }^{3}$ and Katy Martin Rainey ${ }^{1 *}$

\section{Erratum}

Following publication of this article [1], it has come to our attention that some of the equations included were distorted by a formatting issue in the PDF form. This issue affected Eqs. 8, 9 and 11. The correct forms of the equations are shown below.

$$
\begin{aligned}
& \sigma_{\mathrm{b}}^{2} \mid * \sim \frac{\mathbf{b}^{\prime} \mathbf{b}+\mathrm{S}_{\mathrm{b}} v_{\mathrm{b}}}{X_{\mathrm{p}+v_{\mathrm{b}}}^{2}} \text { and } \sigma_{\mathrm{e}}^{2} \mid * \sim \frac{\mathbf{e}^{\prime} \mathbf{e}+\mathrm{S}_{\mathrm{e}} v_{\mathrm{e}}}{X_{\mathrm{n}+v_{\mathrm{e}}}^{2}} \\
& \mathrm{~b}_{\mathrm{j}}^{\mathrm{t}+1} \mid * \sim \mathrm{N}\left(\frac{\widetilde{\mathbf{x}}_{\mathrm{j}}^{\prime} \widetilde{\mathbf{e}}^{\mathrm{t}}+\psi \mathbf{x}_{\mathrm{j}}^{\prime} \mathbf{x}_{\mathrm{j}} \mathrm{b}_{\mathrm{j}}^{\mathrm{t}}}{\psi \mathbf{x}_{\mathrm{j}}^{\prime} \mathbf{x}_{\mathrm{j}}+\lambda_{\mathrm{j}}}, \frac{\sigma_{\mathrm{e}}^{2}}{\psi \mathbf{x}_{\mathrm{j}} \mathbf{x}_{\mathrm{j}}+\lambda_{\mathrm{j}}}\right) \\
& \sigma_{\mathrm{e}}^{2} \mid * \sim \frac{\widetilde{\mathbf{e}}^{\prime} \widetilde{\mathbf{e}}+\mathrm{S}_{\mathrm{e}} v_{\mathrm{e}}}{X_{\psi \mathrm{n}+v_{\mathrm{e}}}^{2}}
\end{aligned}
$$

\section{Author details}

'Department of Agronomy, Purdue University, 915 W. State St., Lilly Hall, West Lafayette, IN 47907, USA. ²Department of Plant Science, University of California, 3134 Batchelor Hall, Riverside, CA 92521, USA. ${ }^{3}$ Department of Animal Science, Purdue University, 915W. State St., Lilly Hall, West Lafayette, IN 47907, USA.

Received: 3 April 2017 Accepted: 7 April 2017

Published online: 26 April 2017

\section{Reference}

1. Xavier A, Xu S, Muir W, Rainey K. Genomic prediction using subsampling. BMC Bioinformatics. 2017;18:1. doi:10.1186/s12859-017-1582-3.

\footnotetext{
* Correspondence: krainey@purdue.edu

'Department of Agronomy, Purdue University, 915 W. State St., Lilly Hall, West Lafayette, IN 47907, USA
} 